\title{
COMPTE-RENDUS DE CONGRES ET REVUE DE LA LITTERATURE INTERNATIONALE
}

avec la collaboration de :

\author{
J. BELAISCH \\ M.A. DROSDOWSKY \\ J.F. GUERIN \\ H. LEJEUNE
}




\section{Compte-rendus de Congrès et Revue de la littérature internationale}

\section{COMPTE-RENDUS DE CONGRES}

389 Sessions «Andrologie » des 2 congrès internationaux concernant la procréation : 10th World Congress on IVF and Assisted Reproduction (Vancouver, 24-28 mai 1997) et 13th Annual Meeting of ESHRE (European Society of Human Reproduction and Embryology, Edimbourg, 22-25 juin 1987). J.F.GUERIN

\section{ENDOCRINOLOGIE}

391 La FSH est nécessaire à la maturation folliculaire mais pas à la fertilité mâle T.R. KUMAR, Y. WANG, N.F. LU, M.M. MATZUK

Nature Genetics, 1977, 15 : 201-204

391 Les hommes homozygotes pour une mutation inactivant le récepteur à la FSH présentent des degrés variables d'altération de la spermatogenèse et de la fertilité J.S. TAPANAINEN, K. AITTOMAKI, J. MIN et al.

Nature Genetics, 1977, $15: 205-206$

394 Comparaison entre la spécificité du ligand et la distribution tissulaire du transcrit des récepteurs aux oestrogènes a et $b$

G. KUIPER, B. CARLSSON, K. GRADIEN et al.

Endocrinology, 1997, 138,3, 863-870

\section{SPERMIOLOGIE}

395 Réexamen d'une métaanalyse de 61 études de la concentration spermatique S. BECKER, K. BERHANE

Fertil. Steril., 1997, 67, 1103-1108

$396 \quad$ Echec de la méthode de swim-up pour la préselection du sexe C.J. SEJONGE, S.P. FLAHERTY, A.M. BARNES et al.

Fertil. Steril., 1997, 67, 1109-114 


\section{COMPTE-RENDUS DE CONGRES}

\author{
Compte-rendu des sessions «Andrologie» des \\ 2 congrès internationaux concernant la \\ procréation : " 10th World Congress on IVF \\ and Assisted Reproduction» (Vancouver, 24- \\ 28 Mai 1997) et 13th Annual Meeting of \\ ESHRE ( $E$ European Society of Human \\ Reproduction and Embryology", Edim- \\ bourg, 22-25 Juin 1997)
}

J.F. GUERIN

Ces 2 importants congrès consacrés à la reproduction, l'un annuel (ESHRE meeting), l'autre bisannuel (World Congress on IVF \& AR), se sont déroulés à 1 mois d'intervalle.

Contrairement à l'usage, l'auteur de ces compterendus a préféré, plutôt que de traiter séparément les 2 manifestations (ce qui aurait conduit à d'inévitables redites, compte tenu des similitudes des sujets traités, parfois par les mêmes auteurs), effectuer une synthèse globale en considérant quelques grands thèmes abordés dans les 2 congrès. Ce rapport n'est nullement exhaustif, car il eût fallu à l'auteur le don de démultiplication, ce qui n'est pas dans ses compétences!

\section{L'ICSI dans le contexte d'azoospermie sécrétoire}

Ces 2 congrès comprenaient de nombreuses sessions consacrées à l'Andrologie, avec des communications de valeur inégale, et parfois contradictoires. Un nombre très important d'entre elles ont traité de l'azoospermie sécrétoire, en relation avec les possibilités nouvelles offertes par l'ICSI. Plusieurs auteurs ont essayé - en vain de prédire la présence de spermatozoïdes dans les biopsies à partir de critères bio-cliniques. Certains auteurs observent une corrélation positive avec le volume testiculaire, et négative avec la valeur de FSH (Madgar et coll.) ; d'autres ne retrouvent pas cette corrélation (Mukaida et coll.). Il a été souligné à plusieurs reprises qu'un blocage au stade méiotique de la lignée germinale, était souvent associé à une valeur normale de FSH. Quels que soient les critères retenus pour étiqueter une azoospermie "sécrétoire", la plu- part des auteurs, dont Van Steirteghem, font des observations concordantes : des spermatozoïdes testiculaires utilisables en ICSI sont retrouvés dans environ la moitié des cas $(60 \%$ pour Silber). On est donc loin de l'optimisme certainement excessif des premières publications sur le sujet. Pour ce qui concerne l'extraction et le traitement des spermatozoïdes testiculaires, Nijs et coll. (Bruxelles) ont évalué l'intérêt de cultiver les spermatozoïdes 24-30 heures avant l'ICSI, ce qui implique d'effectuer la biopsie testiculaire la veille de la ponction ovocytaire. On sait en effet que la majorité des spermatozoïdes est immobile au moment de l'extraction et qu'une certaine mobilité est susceptible d'apparaître dans les 24 heures. Dans son étude, l'auteur a montré que cette acquisition de la mobilité dans le temps s'effectuait au détriment de la vitalité globale de la population spermatique : celle-ci est en moyenne de $92 \%$ initialement, et chute sévèrement au bout de 24 heures. On prend donc un "risque" plus important, si on n'a pas d'autre choix que d'injecter des spermatozoïdes immobiles, en choisissant une durée d'incubation de 24 heures. De fait, une étude clinique effectuée avec groupe témoin par les mêmes auteurs, ne montre pas de différence significative entre les 2 groupes (5 heures vs 30 heures) en termes de grossesses évolutives. Une communication intéressante de l'équipe de Van Steirteghem, a montré l'intérêt d'effectuer une digestion enzymatique par une collagénase de type IV (les résultats étaient moins bons avec une collagénase de type IA), sur le tissu testiculaire, permettant une meilleure récupération des spermatozoïdes. La même équipe a montré que la cryoconservation du tissu testiculaire donnait de meilleurs résultats si le fragment biopsique était dilacéré avant congélation (le cryoprotecteur pénètre difficilement dans la biopsie entière).

Plusieurs auteurs ont présenté des techniques pour identifier de manière fiable les spermatides rondes (SR) dans les biopsies testiculaires : Lee et al (Corée) ont identifié par RT-PCR le transcrit de la protamine 2, dont l'expression est spécifique de la SR : ils en ont retrouvé dans 18 cas sur 33 azoospermies sécrétoires, et les ont utilisées en ICSI : les taux de fécondation "normale " (zygote à 2 pronucléi ou " $\mathrm{PN}^{\text {") }}$ demeurent bas (27\%), tandis que les taux de fécondation anormale à 1 PN (activation ovocytaire) et 3 PN (digynie par non-expulsion du deuxième globule 
polaire) étaient élevés : respectivement $20,5 \%$ et $2 \%$. Une seule naissance (gémellaire) a été obtenu, à partir de 25 transferts. La capacité de ces $S R$ à engendrer des embryons "normaux" et évolutifs reste donc à démontrer. Liu et al (USA) ont sélectionné les SR par hybridation in situ (FISH), permettant d'identifier les cellules à génome haploïde, donc les SR. Ils ont montré que de nombreuses cellules germinales, sélectionnées a priori comme étant des SR à partir de critères de taille et de forme, se sont en fait révélées être diploïdes en FISH, donc être des spermatocytes ou des spermatogonies, voire des cellules sanguines. Et pourtant, Sofikitis (Athènes), l'un des "pères" de l'injection des SR ("ROSNI") a affirmé pouvoir reconnaître sans risque d'erreurs des SR observées en contraste interférentiel (Normaski). L'auteur a injecté 120 ovocytes avec des SR démembranées (stade $\geq 2$ ) et obtenu 52 œufs fécondés, avec 3 grossesses cliniques pour 15 transferts. Quelques essais ont même été effectués en injectant des spermatocytes II et en stimulant secondairement l'ovocyte par des moyens mécaniques (mal précisés) : l'auteur a observé dans 3 cas l'émission d'un troisième globule polaire, correspondant vraisemblablement à l'expulsion du stock chromosomique excédentaire du spermatocyte II (comme cela avait été observé par Yamagimachi chez la souris). Le transfert des cufs ainsi activés aurait abouti à une grossesse évolutive !

Au total néanmoins, l'injection de SR (on n'ose pas parler des spermatocytes II) apparaît faiblement couronnée de succès, et n'a pas fait la preuve de son innocuité. De plus, son intérêt même est remis en cause par certains auteurs faisant autorité, comme Silber, qui affirme n'avoir jamais observé de SR isolées dans une biopsie testiculaire, sans qu'on trouve également des spermatides allongées.

\section{Aspects génétiques de l'infertilité masculi- ne}

De nombreuses études ont concerné la recherche des micro-délétions du bras long du chromosome Y dans les hypofertilités. L'incidence de ces micro-délétions (MD) est variable selon les auteurs : 5 à $20 \%$, dans une revue de synthèse effectuée par Vogt (Allemagne) ; de fait, elle est dépendante de la sonde utilisée. On peut néanmoins dégager certains résultats intéressants. Selva et coll. (France) ont recherché des MD chez 81 hommes infertiles, mais n'en ont pas trouvé chez les patients dont la numération spermatique dépassait 5 millions / ml. Pour Simoni et Nieschlag (Allemagne), ce seuil peut être abaissé à la valeur d'un million / $\mathrm{ml}$. Silber et coll. (USA) ont retrouvé des $\mathrm{MD}$ du gène $\mathrm{DAz}$ chez $13 \%$ de patients azoospermiques $(16 / 125)$ et $5,7 \%$ d'oligozoospermies sévères $(2 / 28)$. Chez les azoospermiques, les taux de biopsies testiculaires positives (pour lesquelles une ICSI a pu être réalisée) étaient respectivement de $54 \%$ $(22 / 41)$ et $37 \%(3 / 8)$, selon que le gène DAz était normal ou muté. Au total, pour les 10 cas où une ICSI a été pratiquée dans un contexte de mutation du gène $\mathrm{DAz}$ ( 8 azoospermies et 2 oligozoospermies), les taux d'implantation par embryon transféré étaient de $14 \%$ (vs $18 \%$ chez les témoins). L'existence de $M D$ du gène $D A z$ ne semble donc pas compromettre les chances de grossesse. Mais le couple devra bien être informé du risque de transmission de l'hypofertilité paternelle, et non uniquement, comme on le croyait jusqu'à une période récente, s'il s'agit d'un garçon. On vient en effet de découvrir un homologue du gène DAz sur un autosome : le chromosome 3 (revue par Vogt). 


\section{ANDOCRINOLOGIE}

La FSH est nécessaire à la maturation folliculaire mais pas à la fertilité mâle.

\section{Follicle stimulating hormone is required for ovarian follicle maturation but not male fertility.}

T.R. KUMAR, Y. WANG, N.F. LU, M.M. MATZUK

Departments of Pathology, Cell Biology and Molecular and Human Genetics, Baylor College of Medicine, Houston,TX 77030 USA

Nature Genetics, 1997, 15 : 201-204.

La FSH appartient à la famille des hormones glycoprotéiques qui comporte aussi la $\mathrm{LH}$, la TSH et la gonadotrophine chorionique. Ces hormones ont en commun une sous-unité a et diffèrent par leur sous-unité b. Seuls les hétérodimères ont une activité biologique. Les récepteurs de la FSH sont localisés sur les cellules de Sertoli dans le testicule et les cellules de la granulosa dans l'ovaire. Pour disposer d'un modèle animal des maladies humaines du mécanisme de transduction du signal des gonadotrophines, des souris transgéniques avec inactivation ciblée du gène de la sous unité b de la FSH ont été générées grâce à la technologie des cellules ES (embryonic stem cells).

Les souris hétérozygotes sont viables et fertiles. Elles ont été croisées entre elles pour obtenir des souris homozygotes déficitaires en FSH. Bien que la FSH soit considérée comme nécessaire à la spermatogénèse et la multiplication des cellules de Sertoli, les souris mâles déficitaires en sous-unité b-FSH sont fertiles. Elles ont toutefois des testicules de petite taille. Histologiquement, il existe une réduction du volume total des tubes séminifères. Qualitativement tous les stades de maturation des cellules germinales sont présents et de nombreux tubules contiennent des spermatozoïdes. Néanmoins, le nombre de spermatozoïdes dans l'épididyme est réduit de $75 \%(0,13 \pm 0,4 \times 106$ vs $5,2 \pm 0,2 \times 106)$, le nombre de spermatozoïdes mobiles est réduit de $40 \%$, par contre la vitalité n'est pas modifiée. Le nombre de cellules de Leydig est augmenté de manière relative sur les coupes histologiques, mais est probablement normal, compte tenu de la petite taille des testicules. La testostérone circulante est normale et les glandes annexes sont normales.

Les souris déficitaires femelles sont infertiles, l'utérus et les ovaires sont petits, il n'y a pas de corps jaune dans les ovaires, on constate un blocage de la maturation folliculaire avant la formation des follicules pré-antraux. Le traitement par les gonadotrophines (PMSG/hCG) de souris de 3 semaines permet d'obtenir le même nombre d'ovocytes et d'embryons pour les souris déficitaires et les souris contrôles.

Ces résultats montrent que la FSH est indispensable à la maturation folliculaire, mais, par contre, non absolument indispensable à la fertilité chez le mâle.

Commentaires (voir article suivant)

$\bullet \bullet \bullet$

Les hommes homozygotes pour une mutation inactivant le récepteur à la FSH présentent des degrés variables d'altération de la spermatogénèse et de la fertilité

Men homozygous for an inactivating mutation of the follicle-stimulating hormone (FSH) receptor gene present variable suppression of spermatogenesis and fertility

J.S. TAPANEINEN K. AITTOMAKI, J. Min, T. VASKIVUO, I.T. HUHTANIEMI

Oulu Univ Hosp, Dept Obstet \& Gynecol, FIN 90220 Oulu, FINLAND

Nature Genetics, 1997, 15 : 205-206.

La fonction gonadique est contrôlée par les deux gonadotrophines hypophysaires LH et FSH. Alors que la $\mathrm{LH}$ régule la stéroïdogénèse. $\mathrm{La}$ FSH est considérée comme essentielle pour la folliculogénèse chez la femme. Chez l'homme, la FSH est en général considérée comme essentielle pour l'initiation de la spermatogénèse à la puberté et le maintien d'une production quantitativement normale de spermatozoïdes chez l'adulte.

Une mutation ponctuelle inactivatrice du gène du récepteur de la FSH a été récemment mise en 
évidence chez 22 femmes, issues de 13 familles finlandaises [1]. Cette mutation provoque, chez les femmes homozygotes, une insuffisance ovarienne périphérique transmise sur un mode autosomique récessif [2]. Cette mutation 566C-$>\mathrm{T}$ induit la substitution d'une alanine en valine, elle est localisée dans l'exon 7 du gène du récepteur à la $\mathrm{FSH}$, dans la région codant pour le domaine extramembranaire. Sur le plan fonctionnel, il existe une nette diminution de l'activité de liaison du récepteur et de la transduction du signal par le récepteur muté. Il apparaît ainsi que cette perte de la fonction de FSH est incompatible avec la maturation du follicule ovarien et la fertilité féminine.

Les femmes porteuses de la mutation ont 25 frères dont 15 ont pu être explorés. Quatre sont indemnes, 6 hétérozygotes et 5 homozygotes pour la mutation, ce qui permet de décrire pour la première fois, des hommes homozygotes pour une mutation inactivatrice du gène du récepteur à la FSH. Tous sont en bonne santé, normalement masculinisés, 2 de ces hommes ont chacun 2 enfants. La seule anomalie clinique est un volume testiculaire plus ou moins nettement inférieur à la normale entre 4 et $15,8 \mathrm{ml}$. Le spermogramme montre la présence de spermatozoïdes pour tous les sujets : trois hommes ont une oligospermie sévère $(<1106 / \mathrm{ml})$, un homme a une oligospermie modérée $(5,6106 / \mathrm{ml})$, le dernier a une concentration normale (42 106/ml) mais un faible volume $(1,5 \mathrm{ml})$ et une tératospermie ( $16 \%$ de formes normales). Tous les sujets ont une concentration plasmatique de FSH élevée et d'inhibin- $B$ basse. La testostérone est maintenue normale au prix d'une LH supérieure à la normale pour 3 sujets.

Pour juger de l'importance de la mutation $566 \mathrm{C}$ $\rightarrow$ T dans l'infertilité masculine, cette mutation a été recherchée dans la région géographique des familles atteintes, chez 151 hommes présentant un trouble de la spermatogénèse sévère ou modéré. Il n'a été trouvé aucun sujet homozygote et seulement 2 sujets hétérozygotes. Ceci suggère que cette mutation n'est pas une cause fréquente d'infertilité.

On note que les parents des sujets homozygotes sont obligatoirement hétérozygotes, et sont fertiles comme le montre la taille élevée de certaines fratries dans les familles atteintes (une famille de 11 enfants et une famille de 9 enfants).
Si les femmes homozygotes pour la mutation 566C-->T inactivatrice du récepteur à la FSH sont stériles, par blocage de la maturation folliculaire, il apparaît que chez l'homme, cette mutation ne diminue la spermatogénèse qu'à des degrés variables. Le caractère complet du blocage de la maturation folliculaire chez la femme, la liaison très faible de la $\mathrm{FSH}$ au récepteur mutant $(<5 \%)$ et l'absence de réponse de l'AMPc dans les cellules transfectées par le récepteur mutant font que l'hypothèse d'un blocage partiel de la fonction du récepteur à la FSH ne peut pas être retenue.

Ces résultats montrent que la FSH n'est pas absolument indispensable à la spermatogénèse, elle joue néanmoins un rôle dans le développement normal du volume testiculaire et d'une spermatogénèse quantitativement normale.

\section{Commentaires (Hervé LEJEUNE)}

Ces 2 articles sont complémentaires et concordants, ils permettent de préciser le rôle de la FSH sur la spermatogénèse, sujet controversé depuis de nombreuses années [3]. L'inactivation ciblée, chez la souris, du gène de la sous-unité bFSH et l'homozygotie, en pathologie humaine, d'une mutation inactivatrice du récepteur à la FSH, représentent deux situations dans lesquelles l'action de la FSH est supprimée de manière spécifique. Les conséquences sont très similaires dans les deux cas. Dans le sexe féminin, le blocage de la maturation folliculaire est total à partir du premier stade précédemment reconnu comme FSH-dépendant (passage aux follicules pré-antraux).

Dans le sexe masculin, les conséquences sont moins massives, la spermatogénèse n'est que partiellement diminuée quantitativement et pas ou peu perturbée sur le plan qualitatif, avec conservation d'une fécondance des spermatozoïdes, tout au moins pour certains d'entre eux, au point que la fertilité soit conservée (les données disponibles ne permettent toutefois pas la quantification de cette fertilité).

Les données de ces deux publications confirment certaines données de la physiologie "classique". La FSH intervient dans la maturation folliculaire à partir du passage au stade pré-antral, on constate ici qu'elle est indispensable. Chez le mâle, le rôle partiel de la FSH dans le maintien de la spermatogénèse chez l'adulte était déjà suggéré aussi bien dans les modèles animaux que 
dans l'espèce humaine. Les manipulations hormonales ont montré que si la maturation testiculaire avait eu lieu normalement et que la concentration intratesticulaire de testostérone était maintenue normale, la spermatogénèse se déroulait normalement sur le plan qualitatif en l'absence de FSH [4]. Dans l'espèce humaine, la synergie entre testostérone intratesticulaire et FSH avait été démontrée, la présence des 2 gonadotrophines, FSH et LH, permettant une spermatogénèse quantitativement normale [5]. La possibilité de grossesse avec des spermatogenèses maintenues à un niveau partiel est d'observation clinique courante, en particulier dans les situations de déficit gonadotrope, en cours de traitement substitutif par les gonadotrophines. Par contre, on pensait que la FSH avait un rôle assez indispensable dans la mise en place de la spermatogénèse. C'est à cet égard que ces 2 articles apportent des notions nouvelles, puisque dans les deux situations, l'inactivation de la FSH est constitutive et que la spermatogénèse se met tout de même en place. La diminution de la taille des testicules chez les souris transgéniques et chez les hommes homozygotes pour la mutation confirme le rôle de la FSH dans la multiplication des cellules de Sertoli [6]. Le nombre de cellules de Sertoli déterminant par la suite l'aspect quantitatif de la spermatogénèse et la taille des testicules à l'âge adulte [7].

On remarque la similitude du tableau induit par l'inactivation spécifique de la FSH ou de son récepteur et avec l'inactivation de la cycline-D2 (commentaire dans le précédent numéro d'Andrologie) qui semble intervenir dans l'effet de la FSH sur la multiplication des cellules de la granulosa et des cellules de Sertoli [8].

Ainsi la FSH représente un facteur non indispensable à la fertilité masculine mais reste un cofacteur participant à la multiplication des cellules de Sertoli et à l'aspect quantitatif de la spermatogénèse.

La notion du caractère non indispensable de la FSH pour qu'une fertilité soit possible remet en cause les possibilités de contraception masculine basées uniquement sur la suppression de la FSH ou de son action.

Les possibilités d'anomalies de la FSH ou de ses récepteurs en pathologie humaine restent ouvertes, mais ces anomalies risquent d'être plus responsables d'oligospermies et d'hypofécondité que d'azoospermie.

\section{REFERENCES}

1. AITTOMAKI K., LUCENA J.L.D., PAKARINEN P. et al. : Mutation in the follicle-stimulating hormone receptor gene causes hereditary hypergonadotropic ovarian failure. Cell, 1995, 82 : 959-968.

2. AITTOMAKI K., HERVA R., STENMAN U.H. et al. : Clinical features of primary ovarian failure caused by a point mutation in the follicle-stimulating hormone receptor gene. J. Clin. Endocrinol. Metab., 1996, 81 : 3722-3726.

3. ZIRKIN B.R., AWONIYI C., GRISWOLD M.D., RUSSELL L.D., SHARPE R. : Minireview: is FSH required for adult spermatogenesis ? J. Androl., 1994, $15: 273-276$.

4. FINKEL DM, PHILLIPS JL, SNYDER PJ : Stimulation of spermatogenesis by gonadotropins in men with hypogonadotropic hypogonadism. N. Engl. J. Med., 1985, 313:615-655

5. BREMNER W.J., MATSUMOTO A.M., SUSSMAN A.M., PAULSEN C.A. : Follicle-stimulating hormone and spermatogenesis. J. Clin. Invest., 1981, 68 : 1044-1052.

6. ORTH J.M. : The role of follicle-stimulating hormone in controling Sertoli cell proliferation in testes of fetal rats. Endocrinology, 1984, 115 : 1248-1255.

7. ORTH J.M., GUNSALUS G.M., LAMPERTI A.A. : Evidence from Sertoli cell-depleted rats indicates that spermatid number in adults depends on Sertoli cells produced during perinatal development. Endocrinology, 1988, $122: 787-794$.

8. SICINSKI P., DONAHER J.L., GENG Y. et al. : Cyclin D2 is an FSH responsive gene involved in gonadal cell proliferation and oncogenesis. Nature, 1996, $384: 470-474$. 
Comparaison entre la spécificité de liaison du ligand et la distribution tissulaire du transcrit des récepteurs aux oestrogènes $\alpha$ et $\beta$

\section{Comparison of the ligand binding specifici-} ty and transcript tissue distribution of estrogen receptors $\alpha$ and $\beta$

G. Kuiper, B. Carlisson, K. Grandien, E. ENMARK, J. HÄGGBLAD, S. NILSSON AND

J.A. GUSTAFSSON

Endocrinology 138, 3, 863-870, 1997.

Les oestrogènes influencent la croissance, la différenciation et le fonctionnement de très nombreux tissus cibles comme la glande mammaire, l'utérus, I'ovaire, le testicule et la prostate. Les oestrogènes jouent aussi un rôle important dans le métabolisme osseux et dans le système cardiovasculaire. On sait depuis longtemps que les composés oestrogèniques se lient au niveau de ces tissus à un récepteur (ER), mais ce n'est que très récemment que Kuiper a mis en évidence deux types de récepteurs Er $\alpha$ et ER $\beta$, ce dernier plus particulièrement au niveau de la prostate.

Le cDNA de ER $\beta$ chez le rat encode une protéine de 485 acides aminés avec un poids moléculaire de 54200 . Cette protéine est très homologue à la protéine ER $\alpha$, en particulier en ce qui concerne le domaine de liaison au DNA $(80 \%$ des acides aminés sont identiques) et dans la partie C-terminale du domaine de liaison du ligand ( $55 \%$ ).

Tant en ce qui concerne ER $\alpha$ que ER $\beta$, la courbe de Scatchard est représentée par une droite, ce qui correspond à un seul type de site de liaison ; néanmoins, l'affinité d'une trentaine de substances à activités plus ou moins oestrogèniques, varie lorsqu'on passe de $\mathrm{ER} \alpha$ à $\mathrm{ER} \beta$.

Pour ER $\alpha$ : diethylstilbestrol > hexestrol > dienestrol $>4$ hydroxy-tamoxifen $>17 \beta$-oestradiol $>$ coumestrol $>$ ICI $164384>$ oestrone, $17 \alpha$ oestradiol $>$ nafoxidine, moxestrol $>$ clomiphene $>$ oestriol, 4-hydroxy-oestriol > tamoxifen, 2 hydroxy-oestradiol, 5 androstene- $3 \beta$, $17 \beta$,diol,genisteine et ce qui concerne ER $\beta$,: dienestrol $>4$ hydroxy-tamoxifen $>$ diethylstilbestrol $>$ hexestrol $>$ coumestrol, ICI $164384>$ $17 \beta$, oestradiol $>$ oestrone, genisteine $>$ oestriol $>$ nafoxidine, 5 androstene-3,17 $\beta$, diol $>17 \alpha$ oestradiol, clomiphene, 2-hydroxy-oestradiol $>4$ hydroxy-oestradiol, tamoxifene, moxestrol.
Si l'affinité est assez semblable en ce qui concerne les ligands physiologiques, une différence intéressante a été notée pour le $17 \alpha$-oestradiol qui a une affinité 5 fois plus grande pour la protéine $\mathrm{ER} \alpha$. Un autre point digne d'intérêt est la répartition des récepteurs $\mathrm{ER} \alpha$ et $\mathrm{ER} \beta$ en fonction des tissus. L'expression la plus importante du mRNA d'ER $\beta$ a été trouvée au niveau de l'ovaire et de la prostate. Une expression moyenne a été déterminée au niveau du testicule, de l'utérus, de la vessie et du poumon alors qu'au niveau de l'hypophyse, de l'épididyme, du thymus, de diverses régions du cerveau et de la moelle épinière, l'expression est faible.

En ce qui concerne maintenant le mRNA d'ERa, I'expression est forte au niveau de l'épididyme, du testicule, de l'hypophyse, de l'utérus, du rein et de la surrénale alors qu'elle est modérée ou nulle pour le mRNA de ER $\beta$,. L'ovaire et l'utérus contiennent les deux protéines.

\section{Commentaires (M.DROSDOWSKY)}

La signification biologique de ces deux récepteurs n'est pas claire en ce moment. Peut-être l'existence de ces deux sous-types apporte une explication pour l'action sélective des oestrogènes au niveau des différents tissus cibles. I1 est cependant curieux de constater que l'homologie du segment $L B D$ (ligand binding domain) ne dépasse pas 55 $\%$.

On s'est aussi posé la question de savoir pourquoi on a tant tardé à mettre ces deux variétés de récepteurs en évidence? La réponse réside dans la faible concentration d'ER et donc la difficulté de le révéler par immunologie alors que la révélation du mRNA est beaucoup plus sensible. 


\section{SPERMIOLOGIE}

\section{Réexamen d'une métaanalyse de 61 études de la concentration spermatique}

\section{A meta analysis of 61 sperm count studies revisited}

S. BECKER, K. BERHANE

John Hopkins University et Pittsburg University, USA

Fertil Steril 1997, $67:$ 1103-1108,

Objectif : réexaminer les données de l'étude sur les concentrations spermatiques provenant du monde entier.

Moyens : analyses paramétriques et modèles non linéaires flexibles de la relation entre la concentration des spermatozoides et le temps.

Principales mesures : concentration spermatique/ml et coefficients de régression pour une possible évolution des concentrations avec les années.

Résultats et conclusions : Aucun déclin général à travers le monde n'a été constaté. Aucune évolution significative n'a été observée pour les pays hors USA, ni par les réanalyses paramétriques, ni par les modèles flexibles non linéaires. Seules les études concernant les USA ont montré un declin significatif.

\section{Commentaires (J BELAISCH):}

Ces commentaires sont rédigés par un andrologue dont l'incompétence en matière de statistiques est absolue. Seules donc les conclusions des études statistiques redigées en clair dans l'article sont utilisées.

La première réflexion est brutale et banale. Cette réanalyse des données de CARLSEN qui ont bouleversé le monde andrologique, aussi bien que la grande presse de vulgarisation, a donné des résultats opposés à ceux du premier travail : il n'y a pas de baisse généralisée à travers le monde. Le déclin n'est observé qu'aux USA.

Au lecteur de décider si c'est la première ou la deuxième analyse qui reflète la réalité des faits.
Deuxième réflexion, le sperme des hommes non américains est significativement plus concentré que celui des américains. Les raisons de cette discordance ne sont pas abordées.

Troisième réflexion, même chez les américains, depuis 1975, il n'y a plus de déclin. L'hypothèse des effets des toxiques industriels semble ainsi écartée car il est peu probable que l'industrie américaine ait régressé depuis cette date. Néanmoins, une plus grande attention aux problèmes d'environnement a peut être aussi exercé ses effets favorables.

Quatrième réflexion, ainsi, il est impossible de distinguer, à partir de ces données, si la vraie pente pour I'Europe était zéro ou au même niveau que la pente pour les US ? Cette traduction littérale de la fin d'un paragraphe de l'article montre sans ambiguité Ia légéreté des données sur lesquelles tant de commentaires, angoissants et angoissés ont été rédigés.

Fait encore plus troublant lorsqu'on additionne, sur le modèle flexible non linéaire, les données US et non US, on observe une remontée depuis 1975. Lorsqu'on les sépare, plus aucune remontée n'apparaît! Les taux sont stables pour les non US et continuent à décliner très faiblement ou à se stabiliser pour les US.

Aucune réflexion n'a été faite dans cet article sur les effets possibles de différentes méthodes de mesure des concentrations spermatiques, ni sur les extraordinaires divergences entre les valeurs trouvées aux USA comme dans les pays non US en 1980. Celles-ci par exemple pour les USA, s'échelonnent entre 50 et 130 millions $/ \mathrm{ml}$. Aucune réflexion non plus sur la curieuse discordance entre les études de AUGER et coll, montrant une baisse récente des concentrations alors que les autres études semblent présenter une remontée et que le déclin pour celles-ci n'avait été observé que dans les années 70 ou début des années 80.

Un autre sujet d'étonnement pour le lecteur non averti tient en cette mise en commun des données qui montrent un déclin comme se prouvant mutuellement alors que les valeurs majeures de départ sont tout à fait différentes et les périodes de déclin contradictoires.

Un exemple de ces discordances a été parfaitement analysé par AUGER, dans les colonnes d'Andrologie ( numéro de mars 1997), qui montrait un accroissement très marqué des lésions 
testiculaires chez les sujets décédés de mort subite, en Finlande, pays qui s'était distingué par la stabilité de ses spermogrammes dans l'étude de VIERULA ! Mais les zones d'étude n'étaient pas les mêmes.

Vis-à-vis des statistiques démontrant le déclin du sperme, les français peuvent se permettrent de suivre leur vieil adage : il leur faut "raison garder".

\section{0}

\section{Echec de la méthode de swim-up pour la préselection du sexe}

\section{Failure of mutitube sperm swim-up for sex preselection}

C.J. SEJonge, S.P. FlaherTy, A.M. BaRnes, N.J. Swann, C. MatThews

Départements d'Obstétrique et Gynécologie du Centre Médical de l'Université d'AdélaïdeAustralie du Nord

Fertil. Steril.,1997, 67, 1109-1114

Objectif : Accroitre les proportions de spermatozoildes (porteurs de l'X ou l'Y) par une méthode de swim-up et en vérifier l'efficacité à l'aide d'une hybridation in situ par sondes spécifiques fluorescentes.

\section{Conditions : double aveugle}

Materiel : patients consultant pour problème de fertilité.

Methodes : étude de deux couches dans lesquelles les spermatozoïdes réunis en pellets lâches par centrifugation (à 300 tours minutes pendant 3 à 5 minutes) sont libres de quitter (ou de réintégrer) les pellets par swim-up. Les multitubes ont pour but de n'obtenir que des pellets peu denses, ce qui facilite l'évasion des spermatozoïdes.

Résultats : Aucune modification cliniquement significative de la proportion des spermatozoïdes $\mathrm{X}$ ou Y n'a été observée dans les deux couches surmontant les pellets et qui étaient censées,d'après un travail précédent de Rawlins et al (cité 5 fois dans le texte in extenso), être enrichies en l'une et l'autre catégories de spermatozoïdes.
Conclusion : l'application clinique de ce procédé doit être fortement découragée.

\section{Commentaires ( $J$ BELAISCH)}

La recherche d'une méthode efficace de préselection du sexe est un objectif poursuivi depuis des années et par de très nombreuses équipes. Aucune ne s'est montrée satisfaisante jusqu'ici, sauf la cytométrie de flux qui, appliquée aux lapins, s'est révélée à l'origine d'une réduction des portées, sans compter les effets mutagènes possibles de la technique.

Apparemment une équipe sous la signature de RAWLINS et al avait annoncé un résultat satisfaisant avec $60 \%$ de spermatozoïdes porteurs d'Y dans la portion enrichie contre $36 \%$ de la portion enrichie en $X$.

Les auteurs qui ont échelonné les temps de centrifugation (15,30,45 et 60 minutes) n'ont trouvé aucun enrichissement sauf lorsque la centrifugation était de 30 minutes. Mais l'accroissement de la proportion de spermatozoïdes Y n'était alors que de 1 à $2 \%$ !

De façon tout à fait inexpliquée (sinon inexplicable) l'équipe mixte austalo-nord-américaine a modifié les conditions de la centrifugation de Rawlins - passant de 200 à 300 tours / minutes-alors qu'elle cherchait à vérifier les assertions émises par ce groupe de chercheurs, sur le swim up en multitubes.

D'un autre côté, la métode de détermination de la présence de chromosomes sexuels, utilisant une sonde spécifique pour chacun des deux chromosomes sexuels, était beaucoup plus valable alors que la quinacrine, utilisée auparavent, s'est révélée capable de colorer les centromères de certains autosomes et non pas seulement le chromosome $Y$.

Enfin, on doit retenir selon les auteurs que jusqu'ici aucune méthode d'enrichissement en spermatozoïdes, d'un type ou l'autre, n'a fait la preuve de son efficacité en clinique, que ce soit après séparation par gradient de percoll ou d'albumine, ou à l'aide des méhodes basées sur le swim up ou la cytométrie de flux.

Mais c'est surtout la conclusion de leur résumé qui doit être mise en exergue : l'application clinique de ce procédé doit être fortement découragée. A vrai dire, c'est la totalité des méthodes de préselection $d u$ sexe applicables aussi bien aux gamètes qu'aux individus, qui doit être fortement découragée tant que des preuves scientifiques valables n'ont pas été apportées de la réalité des effets proclamés quelle que soit la méthode utilisée. 